\title{
Generalized System for Relaxed Cocoercive Mixed Variational Inequalities and Iterative Algorithms in Hilbert Spaces
}

\author{
Shuyi Zhang, Xinqi Guo and Dan Luan
}

\begin{abstract}
The approximate solvability of a generalized system for relaxed cocoercive mixed variational inequality is studied by using the resolvent operator technique. The results presented in this paper extend and improve the main results of Chang et al.[1], He and $\mathrm{Gu}[2]$ and Verma [3, 4].
\end{abstract}

\section{Introduction and Preliminaries}

In this paper, the approximate solvability of a system of nonlinear variational inequalities involving two relaxed cocoercive mappings in Hilbert spaces is studied, based on the convergence of resolvent method.

Let $H$ be a real Hilbert space, whose inner product and norm are denoted by $\langle\cdot, \cdot\rangle$ and $\|\cdot\|$. Let $I$ be the identity mapping on $H$, and $T(\cdot, \cdot), S(\cdot, \cdot)$ : $H \times H \rightarrow H$ be two nonlinear operator. Let $\partial \varphi$ denote the subdifferential of function $\varphi$, where $\varphi: H \rightarrow R \cup\{+\infty\}$ is a proper convex lower semicontinuous function on $\mathrm{H}$. It is well known that the subdifferential $\partial \varphi$ is a maximal monotone operator. consider a systems of nonlinear variational inequalities ( for short, SNVI) as follows: Find $x^{*}, y^{*} \in H$, such that

$$
\left\langle\rho T\left(y^{*}, x^{*}\right)+x^{*}-y^{*}, x-x^{*}\right\rangle+\varphi(x)-\varphi\left(x^{*}\right) \geq 0, \forall x \in H, \rho>0 ;
$$

Key Words: relaxed cocoercive mixed variational inequality, resolvent method, relaxed cocoercive mapping, convergence of resolvent method.

2010 Mathematics Subject Classification: Primary 47H09; Secondary 47J05, 47J25.

Received: April, 2011.

Revised: April, 2011.

Accepted: February, 2012. 


$$
\left\langle\eta S\left(y^{*}, x^{*}\right)+y^{*}-x^{*}, x-y^{*}\right\rangle+\psi(x)-\psi\left(y^{*}\right) \geq 0, \forall x \in H, \eta>0 .
$$

It is easy to know that the SNVI (1.1) and (1.2) is equivalent to the following projection equations:

$$
\begin{aligned}
& x^{*}=J_{\varphi}\left(x^{*}-\rho T\left(y^{*}, x^{*}\right)\right), \rho>0 \\
& y^{*}=J_{\psi}\left(y^{*}-\eta S\left(x^{*}, y^{*}\right)\right), \eta>0,
\end{aligned}
$$

where $J_{\varphi}=(I+\partial \varphi)^{-1}, J_{\psi}=(I+\partial \psi)^{-1}$.

Next we consider some special cases of the problem (1.1) and (1.2).

(I) If $T=S$, then the SNVI (1.1) and (1.2) reduces to the following system of nonlinear variational inequalities: find $x^{*}, y^{*} \in H$ such that

$$
\begin{aligned}
& \left\langle\rho T\left(y^{*}, x^{*}\right)+x^{*}-y^{*}, x-x^{*}\right\rangle+\varphi(x)-\varphi\left(x^{*}\right) \geq 0, \forall x \in H, \rho>0 ; \\
& \left\langle\eta T\left(y^{*}, x^{*}\right)+y^{*}-x^{*}, x-y^{*}\right\rangle+\psi(x)-\psi\left(y^{*}\right) \geq 0, \forall x \in H, \eta>0 .
\end{aligned}
$$

(II) If $\varphi=\psi$, then the SNVI (1.1) and (1.2) reduces to the following system of nonlinear variational inequalities: find $x^{*}, y^{*} \in H$ such that

$$
\begin{aligned}
& \left\langle\rho T\left(y^{*}, x^{*}\right)+x^{*}-y^{*}, x-x^{*}\right\rangle+\varphi(x)-\varphi\left(x^{*}\right) \geq 0, \forall x \in H, \rho>0 ; \\
& \left\langle\eta S\left(y^{*}, x^{*}\right)+y^{*}-x^{*}, x-y^{*}\right\rangle+\varphi(x)-\varphi\left(y^{*}\right) \geq 0, \forall x \in H, \eta>0 .
\end{aligned}
$$

(III) If $T=S, \varphi=\psi$, then the SNVI (1.1) and (1.2) reduces to the following system of nonlinear variational inequalities: find $x^{*}, y^{*} \in H$ such that

$$
\begin{aligned}
& \left\langle\rho T\left(y^{*}, x^{*}\right)+x^{*}-y^{*}, x-x^{*}\right\rangle+\varphi(x)-\varphi\left(x^{*}\right) \geq 0, \forall x \in H, \rho>0 ; \\
& \left\langle\eta T\left(y^{*}, x^{*}\right)+y^{*}-x^{*}, x-y^{*}\right\rangle+\varphi(x)-\varphi\left(y^{*}\right) \geq 0, \forall x \in H, \eta>0 .
\end{aligned}
$$

which was studied by $\mathrm{He}$ and $\mathrm{Gu}$ in [2].

(IV) If $K$ is closed convex set in $H, \psi=\varphi$ and $\varphi(x)=I_{K}(x)$ for all $x \in K$, where $I_{K}$ is the indicator function of $K$ defined by $I_{K}(x)=$ $\{0, x \in K$ $+\infty$, otherwise , then the SNVI
lowing SNVI: find $x^{*}, y^{*} \in K$ such that

$$
\begin{aligned}
& \left\langle\rho T\left(y^{*}, x^{*}\right)+x^{*}-y^{*}, x-x^{*}\right\rangle \geq 0, \forall x \in K, \rho>0 ; \\
& \left\langle\eta T\left(y^{*}, x^{*}\right)+y^{*}-x^{*}, x-y^{*}\right\rangle \geq 0, \forall x \in K, \eta>0 .
\end{aligned}
$$

The problem (1.9) and (1.10) have been studied by Chang et al. (see [1]).

(V) If $T, S: H \rightarrow H$ are univariate mappings, then the SNVI (1.1) and (1.2) is collapsed to the following SNVI: find $x^{*}, y^{*} \in H$ such that

$$
\left\langle\rho T\left(y^{*}\right)+x^{*}-y^{*}, x-x^{*}\right\rangle+\varphi(x)-\varphi\left(x^{*}\right) \geq 0, \forall x \in H, \rho>0 ;
$$




$$
\left\langle\eta S\left(x^{*}\right)+y^{*}-x^{*}, x-y^{*}\right\rangle+\psi(x)-\psi\left(y^{*}\right) \geq 0, \forall x \in H, \eta>0 .
$$

Further, if $K$ is closed convex set in $H, S=T, \psi=\varphi$ and $\varphi(x)=I_{K}(x)$ for all $x \in K$, where $I_{K}$ is the indicator function of $K$, then the SNVI (1.11) and (1.12) is equivalent to the following SNVI: find $x^{*}, y^{*} \in K$ such that

$$
\begin{aligned}
& \left\langle\rho T\left(y^{*}\right)+x^{*}-y^{*}, x-x^{*}\right\rangle \geq 0, \forall x \in K, \rho>0 \\
& \left\langle\eta T\left(x^{*}\right)+y^{*}-x^{*}, x-y^{*}\right\rangle \geq 0, \forall x \in K, \eta>0,
\end{aligned}
$$

which was studied by Verma in [3].

The following definitions and lemma are needed in the sequel.

\section{Definition 1.1.}

(i) A mapping $T: H \rightarrow H$ is called $r$-strongly monotone, if for each $x, y \in H$, we have

$$
\langle T(x)-T(y), x-y\rangle \geq r\|x-y\|^{2}
$$

for a constant $r>0$. This implies that

$$
\|T x-T y\| \geq r\|x-y\|,
$$

that is, $T$ is $r$-expansive and when $r=1$, it is expansive.

(ii) A mapping $T: H \rightarrow H$ is called $\mu$-cocoercive, if there exists a constant $\mu>0$ such that

$$
\langle T(x)-T(y), x-y\rangle \geq \mu\|T(x)-T(y)\|^{2}, \forall x, y \in H .
$$

Clearly, every $\mu$-cocoercive mapping $T$ is $\frac{1}{\mu}$ - Lipschitz continuous.

(iii) A mapping $T: H \rightarrow H$ is said to relaxed $\gamma$-cocoercive, if there exists a constant $\gamma>0$ such that

$$
\langle T(x)-T(y), x-y\rangle \geq-\gamma\|T(x)-T(y)\|^{2} .
$$

(iv) $T: H \rightarrow H$ is said to be relaxed $(\gamma, r)$-cocoercive, if there exists constants $\gamma, r>0$ such that

$$
\langle T(x)-T(y), x-y\rangle \geq-\gamma\|T(x)-T(y)\|^{2}+r\|x-y\|^{2}, \forall x, y \in H .
$$

Remark 1.1. It follows from the above definitions that a $r$-strongly monotone mapping must be a relaxed $(\gamma, r)$-cocoercive mapping for $\gamma=0$, but the converse is not true. therefore the class of the relaxed $(\gamma, r)$-cocoercive mappings is more general class. 


\section{Definition 1.2.}

(1) A two-variable mapping $T: H \times H \rightarrow H$ is said to be relaxed $(\gamma, r)$ cocoercive, if there exist constant $\gamma, r>0$ such that

$\langle T(x, u)-T(y, v), x-y\rangle \geq-\gamma\|T(x, u)-T(y, v)\|^{2}+r\|x-y\|^{2}, \forall x, y, u, v \in H$.

(2) A mapping $T: H \times H \rightarrow H$ is said to be $\mu$-Lipschitz continuous in the first variable, if there exists a constant $\mu>0$ such that

$$
\|T(x, u)-T(y, v)\| \leq \mu\|x-y\|, \forall x, y, u, v \in H .
$$

Lemma 1.1. Suppose that $\left\{a_{n}\right\},\left\{b_{n}\right\}$ and $\left\{c_{n}\right\}$ are nonnegative sequence satisfying the following inequality

$$
a_{n+1} \leq\left(1-t_{n}\right) a_{n}+b_{n}+c_{n}, n \geq 0,
$$

where $t_{n} \in(0,1), \sum_{n=0}^{\infty} t_{n}=\infty, b_{n}=o\left(t_{n}\right), \sum_{n=0}^{\infty} c_{n}<\infty$, then $\lim _{n \rightarrow \infty} a_{n}=0$.

\section{Algorithms}

In this section, the general two-step models for approximate solutions to the SNVI (1.1) and (1.2) are given.

Algorithm 2.1. For arbitrary chosen initial points $x_{0}, y_{0} \in H$ compute the sequences $\left\{x_{n}\right\}$ and $\left\{y_{n}\right\}$ such that

$$
\left\{\begin{array}{l}
x_{n+1}=\left(1-\alpha_{n}-\delta_{n}\right) x_{n}+\alpha_{n} J_{\varphi}\left(y_{n}-\rho T\left(y_{n}, x_{n}\right)\right)+\delta_{n} u_{n} \\
y_{n}=\left(1-\beta_{n}-\lambda_{n}\right) x_{n}+\beta_{n} J_{\psi}\left(x_{n}-\eta S\left(x_{n}, y_{n}\right)\right)+\lambda_{n} v_{n},
\end{array}\right.
$$

where $J_{\varphi}=(I+\partial \varphi)^{-1}, J_{\psi}=(I+\partial \psi)^{-1}, \rho$ and $\eta>0$ are constants and $\left\{\alpha_{n}\right\},\left\{\beta_{n}\right\},\left\{\lambda_{n}\right\},\left\{\delta_{n}\right\}$ are sequences in $[0,1]$ and $\left\{u_{n}\right\},\left\{v_{n}\right\}$ are bounded sequences in $H$.

If $S=T$, then Algorithm 2.1 is reduced to the following:

Algorithm 2.2. For arbitrary chosen initial points $x_{0}, y_{0} \in H$ compute the sequences $\left\{x_{n}\right\}$ and $\left\{y_{n}\right\}$ such that

$$
\left\{\begin{array}{l}
x_{n+1}=\left(1-\alpha_{n}-\delta_{n}\right) x_{n}+\alpha_{n} J_{\varphi}\left(y_{n}-\rho T\left(y_{n}, x_{n}\right)\right)+\delta_{n} u_{n} \\
y_{n}=\left(1-\beta_{n}-\lambda_{n}\right) x_{n}+\beta_{n} J_{\psi}\left(x_{n}-\eta T\left(x_{n}, y_{n}\right)\right)+\lambda_{n} v_{n}
\end{array}\right.
$$

where $J_{\varphi}=(I+\partial \varphi)^{-1}, J_{\psi}=(I+\partial \psi)^{-1}, \rho$ and $\eta>0$ are constants and $\left\{\alpha_{n}\right\},\left\{\beta_{n}\right\},\left\{\lambda_{n}\right\},\left\{\delta_{n}\right\}$ are sequences in $[0,1]$ and $\left\{u_{n}\right\},\left\{v_{n}\right\}$ are bounded sequences in $H$. 
If $\psi=\varphi$, then Algorithm 2.1 is reduced to the following:

Algorithm 2.3. For arbitrary chosen initial points $x_{0}, y_{0} \in H$ compute the sequences $\left\{x_{n}\right\}$ and $\left\{y_{n}\right\}$ such that

$$
\left\{\begin{array}{l}
x_{n+1}=\left(1-\alpha_{n}-\delta_{n}\right) x_{n}+\alpha_{n} J_{\varphi}\left(y_{n}-\rho T\left(y_{n}, x_{n}\right)\right)+\delta_{n} u_{n} \\
y_{n}=\left(1-\beta_{n}-\lambda_{n}\right) x_{n}+\beta_{n} J_{\varphi}\left(x_{n}-\eta S\left(x_{n}, y_{n}\right)\right)+\lambda_{n} v_{n}
\end{array}\right.
$$

where $J_{\varphi}=(I+\partial \varphi)^{-1}, \rho$ and $\eta>0$ are constants and $\left\{\alpha_{n}\right\},\left\{\beta_{n}\right\},\left\{\lambda_{n}\right\},\left\{\delta_{n}\right\}$ are sequences in $[0,1]$ and $\left\{u_{n}\right\},\left\{v_{n}\right\}$ are bounded sequences in $H$.

If $S=T, \psi=\varphi$, then Algorithm 2.1 is reduced to the following:

Algorithm 2.4. For arbitrary chosen initial points $x_{0}, y_{0} \in H$ compute the sequences $\left\{x_{n}\right\}$ and $\left\{y_{n}\right\}$ such that

$$
\left\{\begin{array}{l}
x_{n+1}=\left(1-\alpha_{n}-\delta_{n}\right) x_{n}+\alpha_{n} J_{\varphi}\left(y_{n}-\rho T\left(y_{n}, x_{n}\right)\right)+\delta_{n} u_{n} \\
y_{n}=\left(1-\beta_{n}-\lambda_{n}\right) x_{n}+\beta_{n} J_{\varphi}\left(x_{n}-\eta T\left(x_{n}, y_{n}\right)\right)+\lambda_{n} v_{n}
\end{array}\right.
$$

where $J_{\varphi}=(I+\partial \varphi)^{-1}, \rho$ and $\eta>0$ are constants and $\left\{\alpha_{n}\right\},\left\{\beta_{n}\right\},\left\{\lambda_{n}\right\},\left\{\delta_{n}\right\}$ are sequences in $[0,1]$ and $\left\{u_{n}\right\},\left\{v_{n}\right\}$ are bounded sequences in $H$.

\section{Main Results}

Based on Algorithm 2.1, the approximation solvability of the SNVI (1.1) and (1.2) is presented.

Theorem 3.1. Let $H$ be a real Hilbert spaces. Let $T(\cdot, \cdot): H \times H \rightarrow H$ be two-variable relaxed $\left(\gamma_{1}, r_{1}\right)$-cocoercive and $\mu_{1}$-Lipschitz continuous in the first variable; $S(\cdot, \cdot): H \times H \rightarrow H$ be two-variable relaxed $\left(\gamma_{2}, r_{2}\right)$-cocoercive and $\mu_{2}$-Lipschitz continuous in the first variable. Suppose that $\left(x^{*}, y^{*}\right) \in H \times H$ is a solution of the problem (1.1) and (1.2) and that $\left\{x_{n}\right\},\left\{y_{n}\right\}$ are the sequences generated by Algorithm 2.1. If $\left\{\alpha_{n}\right\},\left\{\beta_{n}\right\},\left\{\lambda_{n}\right\}$ and $\left\{\delta_{n}\right\}$ are four sequences in $[0,1]$ satisfying the following conditions

(i) $\sum_{n=0}^{\infty} \alpha_{n}=\infty, \sum_{n=0}^{\infty} \delta_{n}<\infty$,

(ii) $\lim _{n \rightarrow \infty}\left(1-\beta_{n}\right)=0, \lambda_{n}=o\left(\alpha_{n}\right)$,

(iii) $0<\rho<\frac{2\left(r_{1}-\gamma_{1} \mu_{1}^{2}\right)}{\mu_{1}^{2}}, 0<\eta<\frac{2\left(r_{2}-\gamma_{2} \mu_{2}^{2}\right)}{\mu_{2}^{2}}$,

(iv) $r_{i}>\gamma_{i} \mu_{i}^{2}, i=1,2$, then the sequences $\left\{x_{n}\right\}$ and $\left\{y_{n}\right\}$ converges strongly to $x^{*}$ and $y^{*}$, respectively. 
Proof. Since $x^{*}$ and $y^{*}$ are a solution to the SNVI (1.1) and (1.2), then

$$
\begin{aligned}
& x^{*}=J_{\varphi}\left(x^{*}-\rho T\left(y^{*}, x^{*}\right)\right), \rho>0 \\
& y^{*}=J_{\psi}\left(y^{*}-\eta S\left(x^{*}, y^{*}\right)\right), \eta>0,
\end{aligned}
$$

It follows from (2.1) that

$$
\begin{aligned}
\left\|x_{n+1}-x^{*}\right\| & =\|\left(1-\alpha_{n}-\delta_{n}\right) x_{n}+\alpha_{n} J_{\varphi}\left(y_{n}-\rho T\left(y_{n}, x_{n}\right)\right) \\
& -\left(1-\alpha_{n}-\delta_{n}\right) x^{*}-\alpha_{n} J_{\varphi}\left(y^{*}-\rho T\left(y^{*}, x^{*}\right)\right)+\delta_{n} u_{n}-x^{*} \delta_{n} \| \\
& \leq\left(1-\alpha_{n}-\delta_{n}\right)\left\|x_{n}-x^{*}\right\|+\delta_{n}\left(\left\|u_{n}\right\|+\left\|x^{*}\right\|\right) \\
& +\alpha_{n}\left\|y_{n}-y^{*}-\rho\left(T\left(y_{n}, x_{n}\right)-T\left(y^{*}, x^{*}\right)\right)\right\| .
\end{aligned}
$$

From the relaxed $\left(\gamma_{1}, r_{1}\right)$ cocoercive and $\mu_{1}$-Lipschitz continuity in the first variable on $T$, we have

$$
\begin{aligned}
& \left\|y_{n}-y^{*}-\rho\left(T\left(y_{n}, x_{n}\right)-T\left(y^{*}, x^{*}\right)\right)\right\|^{2} \\
= & \left\|y_{n}-y^{*}\right\|^{2}-2 \rho\left\langle T\left(y_{n}, x_{n}\right)-T\left(y^{*}, x^{*}\right), y_{n}-y^{*}\right\rangle \\
+ & \rho^{2}\left\|T\left(y_{n}, x_{n}\right)-T\left(y^{*}, x^{*}\right)\right\|^{2} \\
\leq & \left\|y_{n}-y^{*}\right\|^{2}+\rho^{2} \mu_{1}^{2}\left\|y_{n}-y^{*}\right\|^{2}-2 \rho r_{1}\left\|y_{n}-y^{*}\right\|^{2} \\
+ & 2 \rho \gamma_{1}\left\|T\left(y_{n}, x_{n}\right)-T\left(y^{*}, x^{*}\right)\right\|^{2} \\
\leq & \left(1+\rho^{2} \mu_{1}^{2}-2 \rho r_{1}+2 \rho \gamma_{1} \mu_{1}^{2}\right)\left\|y_{n}-y^{*}\right\|^{2} .
\end{aligned}
$$

Substituting (3.2) into (3.1) and simplifying the result, we have

$\left\|x_{n+1}-x^{*}\right\|=\left(1-\alpha_{n}-\delta_{n}\right)\left\|x_{n}-x^{*}\right\|+\theta_{1} \alpha_{n}\left\|y_{n}-y^{*}\right\|+\delta_{n}\left(\left\|u_{n}\right\|+\left\|x^{*}\right\|\right)$.

where $\theta_{1}=\sqrt{1+\rho^{2} \mu_{1}^{2}-2 \rho r_{1}+2 \rho \gamma_{1} \mu_{1}^{2}}<1$ by Condition (iii).

Now we make an estimation for $\left\|y_{n}-y^{*}\right\|$. It follows from (2.1) that

$$
\begin{aligned}
& \left\|y_{n}-y^{*}\right\| \\
= & \|\left(1-\beta_{n}-\lambda_{n}\right) x_{n}+\beta_{n} J_{\psi}\left(x_{n}-\eta S\left(x_{n}, y_{n}\right)\right) \\
- & \left(1-\beta_{n}-\lambda_{n}\right) y^{*}-\beta_{n} J_{\psi}\left(y^{*}-\eta S\left(x^{*}, y^{*}\right)\right)+\lambda_{n} v_{n}-y^{*} \lambda_{n} \| \\
\leq & \left(1-\beta_{n}-\lambda_{n}\right)\left\|x_{n}-y^{*}\right\|+\beta_{n}\left\|x_{n}-x^{*}-\eta\right\| S\left(x_{n}, y_{n}\right)-S\left(x^{*}, y^{*}\right) \| \\
+ & \lambda_{n}\left(\left\|v_{n}\right\|+\left\|y^{*}\right\|\right) \\
\leq & \left(1-\beta_{n}-\lambda_{n}\right)\left\|x_{n}-x^{*}\right\|+\left(1-\beta_{n}-\lambda_{n}\right)\left\|x^{*}-y^{*}\right\| \\
+ & \beta_{n}\left\|x_{n}-x^{*}-\eta\left[S\left(x_{n}, y_{n}\right)-S\left(x^{*}, y^{*}\right)\right]\right\|+\lambda_{n}\left(\left\|v_{n}\right\|+\left\|y^{*}\right\|\right) .
\end{aligned}
$$

Next we estimate $\left\|x_{n}-x^{*}-\eta\left[S\left(x_{n}, y_{n}\right)-S\left(x^{*}, y^{*}\right)\right]\right\|$. From the relaxed $\left(\gamma_{2}, r_{2}\right)$ cocoercive and $\mu_{2}$-Lipschitz cocoercive in the first variable on $S$, we 
get

$$
\begin{aligned}
& \left\|x_{n}-x^{*}-\eta\left[S\left(x_{n}, y_{n}\right)-S\left(x^{*}, y^{*}\right)\right]\right\|^{2} \\
= & \left\|x_{n}-x^{*}\right\|^{2}-2 \eta\left\langle S\left(x_{n}, y_{n}\right)-S\left(x^{*}, y^{*}\right), x_{n}-x^{*}\right\rangle \\
+ & \eta^{2}\left\|S\left(x_{n}, y_{n}\right)-S\left(x^{*}, y^{*}\right)\right\|^{2} \\
\leq & \left\|x_{n}-x^{*}\right\|^{2}+\eta^{2} \mu_{2}^{2}\left\|x_{n}-x^{*}\right\|^{2}+2 \eta \gamma_{2}\left\|S\left(x_{n}, y_{n}\right)-S\left(x^{*}, y^{*}\right)\right\|^{2} \\
- & 2 \eta r_{2}\left\|x_{n}-x^{*}\right\|^{2} \\
\leq & \left(1+\eta^{2} \gamma_{2}^{2}-2 \eta r+2 \eta \gamma_{2} \mu_{2}^{2}\right)\left\|x_{n}-x^{*}\right\|^{2} .
\end{aligned}
$$

Let $\theta_{2}=\sqrt{1+\eta^{2} \gamma_{2}^{2}-2 \eta r_{2}+2 \eta \gamma_{2} \mu_{2}^{2}}<1$ by Condition (iii). Substituting (3.5) into (3.4), we have

$$
\begin{aligned}
\left\|y_{n}-y^{*}\right\| & \leq\left(1-\beta_{n}-\lambda_{n}\right)\left\|x_{n}-x^{*}\right\|+\left(1-\beta_{n}-\lambda_{n}\right)\left\|x^{*}-y^{*}\right\| \\
& +\beta_{n} \theta_{2}\left\|x_{n}-x^{*}\right\|+\lambda_{n}\left(\left\|v_{n}\right\|+\left\|y^{*}\right\|\right) .
\end{aligned}
$$

Combining (3.6) and (3.3), we obtain that

$$
\begin{aligned}
& \left\|x_{n+1}-x^{*}\right\| \\
= & \left(1-\alpha_{n}-\delta_{n}\right)\left\|x_{n}-x^{*}\right\|+\theta_{1} \alpha_{n}\left\|y_{n}-y^{*}\right\|+\delta_{n}\left(\left\|u_{n}\right\|+\left\|x^{*}\right\|\right) \\
\leq & \left(1-\alpha_{n}-\delta_{n}\right)\left\|x_{n}-x^{*}\right\|+\theta_{1} \alpha_{n}\left[\left(1-\beta_{n}-\lambda_{n}\right)\left\|x_{n}-x^{*}\right\|\right. \\
+ & \left(1-\beta_{n}-\lambda_{n}\right)\left\|x^{*}-y^{*}\right\| \\
+ & \left.\beta_{n} \theta_{2}\left\|x_{n}-x^{*}\right\|+\lambda_{n}\left(\left\|v_{n}\right\|+\left\|y^{*}\right\|\right)\right]+\delta_{n}\left(\left\|u_{n}\right\|+\left\|x^{*}\right\|\right) \\
\leq & \left(1-\left(1-\theta_{1}\right) \alpha_{n}\right)\left\|x_{n}-x^{*}\right\|+\alpha_{n}\left[\left(1-\beta_{n}-\lambda_{n}\right)\left\|x^{*}-y^{*}\right\|\right. \\
+ & \left.\lambda_{n}\left(\left\|v_{n}\right\|+\left\|y^{*}\right\|\right)\right]+\delta_{n}\left(\left\|u_{n}\right\|+\left\|x^{*}\right\|\right) .
\end{aligned}
$$

Set $a_{n}=\left\|x_{n}-x^{*}\right\|, t_{n}=\left(1-\theta_{1}\right) \alpha_{n}, b_{n}=\alpha_{n}\left[\left(1-\beta_{n}-\lambda_{n}\right)\left\|x^{*}-y^{*}\right\|+\lambda_{n}\left(\left\|v_{n}\right\|+\right.\right.$ $\left.\left.\left\|y^{*}\right\|\right)\right]$ and $c_{n}=\delta_{n}\left(\left\|u_{n}\right\|+\left\|x^{*}\right\|\right)$ in (3.7). By Lemma 1.1 ensures that $x_{n} \rightarrow x^{*}$ as $n \rightarrow \infty$. This completes the proof.

Remark 3.2. Theorem 2.1 extends and improves the main results of [1], [2], [3] and [4], respectively.

The following theorems can be obtained from Theorem 3.1 immediately.

Theorem 3.3. Let $H$ be a real Hilbert spaces. Let $T(\cdot, \cdot): H \times H \rightarrow H$ be two-variable relaxed $\left(\gamma_{1}, r_{1}\right)$-cocoercive and $\mu_{1}$-Lipschitz continuous in the first variable. Suppose that $\left(x^{*}, y^{*}\right) \in H \times H$ is a solution of the problem (1.3) and (1.4) and that $\left\{x_{n}\right\},\left\{y_{n}\right\}$ are the sequences generated by Algorithm 2.2. If $\left\{\alpha_{n}\right\},\left\{\beta_{n}\right\},\left\{\lambda_{n}\right\}$ and $\left\{\delta_{n}\right\}$ are four sequences in [0,1] satisfying the following conditions

(i) $\sum_{n=0}^{\infty} \alpha_{n}=\infty, \sum_{n=0}^{\infty} \delta_{n}<\infty$, 
(ii) $\lim _{n \rightarrow \infty}\left(1-\beta_{n}\right)=0, \lambda_{n}=o\left(\alpha_{n}\right)$,

(iii) $0<\rho<\frac{2\left(r_{1}-\gamma_{1} \mu_{1}^{2}\right)}{\mu_{1}^{2}}, 0<\eta<\frac{2\left(r_{2}-\gamma_{2} \mu_{2}^{2}\right)}{\mu_{2}^{2}}$,

(iv) $r_{i}>\gamma_{i} \mu_{i}^{2}, i=1,2$, then the sequences $\left\{x_{n}\right\}$ and $\left\{y_{n}\right\}$ converges strongly to $x^{*}$ and $y^{*}$, respectively.

Theorem 3.4. Let $H$ be a real Hilbert spaces. Let $T(\cdot, \cdot): H \times H \rightarrow H$ be two-variable relaxed $\left(\gamma_{1}, r_{1}\right)$-cocoercive and $\mu_{1}$-Lipschitz continuous in the first variable; $S(\cdot, \cdot): H \times H \rightarrow H$ be two-variable relaxed $\left(\gamma_{2}, r_{2}\right)$-cocoercive and $\mu_{2}$-Lipschitz continuous in the first variable. Suppose that $\left(x^{*}, y^{*}\right) \in H \times H$ is a solution of the problem (1.5) and (1.6) and that $\left\{x_{n}\right\},\left\{y_{n}\right\}$ are the sequences generated by Algorithm 2.3. If $\left\{\alpha_{n}\right\},\left\{\beta_{n}\right\},\left\{\lambda_{n}\right\}$ and $\left\{\delta_{n}\right\}$ are four sequences in $[0,1]$ satisfying the following conditions

(i) $\sum_{n=0}^{\infty} \alpha_{n}=\infty, \sum_{n=0}^{\infty} \delta_{n}<\infty$,

(ii) $\lim _{n \rightarrow \infty}\left(1-\beta_{n}\right)=0, \lambda_{n}=o\left(\alpha_{n}\right)$,

(iii) $0<\rho<\frac{2\left(r_{1}-\gamma_{1} \mu_{1}^{2}\right)}{\mu_{1}^{2}}, 0<\eta<\frac{2\left(r_{2}-\gamma_{2} \mu_{2}^{2}\right)}{\mu_{2}^{2}}$,

(iv) $r_{i}>\gamma_{i} \mu_{i}^{2}, i=1,2$, then the sequences $\left\{x_{n}\right\}$ and $\left\{y_{n}\right\}$ converges strongly to $x^{*}$ and $y^{*}$, respectively.

Theorem 3.5. Let $H$ be a real Hilbert spaces. Let $T(\cdot, \cdot): H \times H \rightarrow H$ be two-variable relaxed $\left(\gamma_{1}, r_{1}\right)$-cocoercive and $\mu_{1}$-Lipschitz continuous in the first variable. Suppose that $\left(x^{*}, y^{*}\right) \in H \times H$ is a solution of the problem (1.7) and (1.8) and that $\left\{x_{n}\right\},\left\{y_{n}\right\}$ are the sequences generated by Algorithm 2.4. If $\left\{\alpha_{n}\right\},\left\{\beta_{n}\right\},\left\{\lambda_{n}\right\}$ and $\left\{\delta_{n}\right\}$ are four sequences in $[0,1]$ satisfying the following conditions

(i) $\sum_{n=0}^{\infty} \alpha_{n}=\infty, \sum_{n=0}^{\infty} \delta_{n}<\infty$,

(ii) $\lim _{n \rightarrow \infty}\left(1-\beta_{n}\right)=0, \lambda_{n}=o\left(\alpha_{n}\right)$,

(iii) $0<\rho<\frac{2\left(r_{1}-\gamma_{1} \mu_{1}^{2}\right)}{\mu_{1}^{2}}, 0<\eta<\frac{2\left(r_{2}-\gamma_{2} \mu_{2}^{2}\right)}{\mu_{2}^{2}}$,

(iv) $r_{i}>\gamma_{i} \mu_{i}^{2}, i=1,2$, then the sequences $\left\{x_{n}\right\}$ and $\left\{y_{n}\right\}$ converges strongly to $x^{*}$ and $y^{*}$, respectively.

\section{References}

[1] S. S. Chang, H.W. Joseph Lee, C.K. Chan, Generalized system for relaxed cocoercive variational inequalities in Hilbert spaces, Appl. Math. Letter, 20 (2007), 329-334. 
[2] Z. H He, F. Gu, Generalized system for relaxed cocoercive mixed variational inequalities in Hilbert spaces, Appl. Math. and Comput. 214 (2009), 26-30

[3] R. U. Verma, General convergence analysis for two-step projection methods and applications to variational problems, Appl. Math. Letter, 18 (2005), 1286-1292.

[4] R. U. Verma, Generalized system for relaxed cocoercive variational inequalities and its projection methods, J. Optim.Theory Appl. 121(1)(2004), 203-210.

Shuyi Zhang,

Department of Mathematics,

BoHai University,

Jinzhou, Liaoning, 121013, China.

Email: jzzhangshuyi@126. com

Xinqi Guo,

Dalian City No.37 Middle School,

Dalian, 116011, China.

Email: libby27@163. com

Dan Luan,

Department of Mathematics,

BoHai University,

Jinzhou, Liaoning, 121013, China.

Email: lnluandan@126. com 